\title{
Prevalence of Molecular Subtypes of Breast Carcinoma and Its Comparison between Two Different Age Groups: A Retrospective Study from a Tertiary Care Center of Northeast India
}

Jagannath Dev Sharma ${ }^{1}$ Sachin Khanna ${ }^{2}$ Shubhra Ramchandani ${ }^{3} \quad$ Lopa Mudra Kakoti $^{1} \quad$ Argha Baruah $^{1}$ Vinay Mamidala²

${ }^{1}$ Department of Pathology, BBCl, Guwahati, India

2Department of Surgical Oncology, BBCl, Guwahati, India

${ }^{3}$ Department of Pathology, GNRC, North Guwahati, India
Address for correspondence Shubhra Ramchandani, MD, Hemoprabha Apartments, Gopinath Nagar, Sankrapura, Guwahati 781016, India (e-mail: shubhraramchandani16@gmail.com).

South Asian J Cancer 2021;10:220-224.

\author{
Abstract

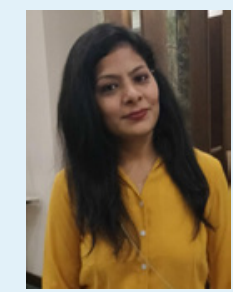 \\ Shubhra Ramchandani \\ Keywords \\ - estrogen receptor \\ - progesterone receptor \\ - human epidermal \\ growth factor \\ - luminal A \\ - luminal B \\ - triple negative
}

Objective The aim of the study is to see the prevalence of different molecular subtypes in breast cancer patients among two different age groups: $\leq 40$ years and $>40$ years.

Materials and Methods Retrospective study was conducted from January 2019 to December 2019. We studied 568 cases of breast carcinoma and classified them into four molecular subtypes-luminal A, luminal B, human epidermal growth factor-2 (HER 2), and triple negative. Cases were divided into two different groups: ( 1$) \leq 40$ years and $(2)>40$ years.

Statistical Analysis was done by using SPSS software version 20.0.

Results Out of 568 cases, 151 (26.6\%) were $\leq 40$ years of age and 417 (73.4\%) were $>40$ years of age. The most common histological subtype of breast cancer was ductal carcinoma in 548 cases and the most common grade was grade III. Immunohistochemistry was done in 432 patients. In younger age group, the most common molecular subtype was luminal B (31\%) followed by triple negative (20\%), luminal $A(14 \%)$, and then HER 2 (5.3\%), while in the older age group most common molecular subtype was luminal B (27.8\%) followed by triple negative (14\%), HER 2 (12.2\%), and then luminal $A(12 \%)$.

Conclusion Luminal B is found to be the most common subtype in Northeast Indian women with breast cancer, as compared with other studies in which luminal A was the most common subtype. This could be due to the reason that Ki 67 was not done in most of the other studies. Comparison between Two Different Age Groups: A Retrospective Cancer 2021;10(4):220-224
C 2021. MedIntel Services Pvt Ltd.

This is an open access article published by Thieme under the terms of the Creative Commons Attribution-NonDerivative-NonCommercial-License, permitting copying and reproduction so long as the original work is given appropriate credit. Contents may not be used for commercial purposes, or adapted, remixed, transformed or built upon. (https://creativecommons.org/licenses/by-nc-nd/4.0/).

Thieme Medical and Scientific Publishers Private Ltd A-12, Second Floor, Sector -2, NOIDA -201301, India 


\section{Introduction}

Breast cancer accounts for the most common cancer in women worldwide. It is multifactorial with both genetic as well as environmental factors playing a role in carcinogenesis. The various risk factors include: age, family history, marital status, menstrual history, hormonal exposure, and lifestyle. ${ }^{1,2}$ Breast cancer in young women is increasing in Asia due to their western lifestyle. ${ }^{3}$

Risk factors, prognosis, and tumor biology are different in younger age group ( $\leq 40$ years) than the older group (>40 years) suggesting it represents different entity. ${ }^{1,4}$ Breast cancer in young women is more aggressive with higher mortality and recurrence rate as compared with older women, however, incidence is more common in older women. ${ }^{5}$

Prognosis depends on-histological type, grade, lymph node metastasis, hormonal receptor status-(estrogen receptor [ER], progesterone receptor [PR], human epidermal growth factor receptor [HER 2]), and proliferation index (Ki 67).,1,46

Breast cancer has varied clinical and molecular characteristics. It can be divided into five molecular groups: luminal A, luminal B, HER 2, basal and normal like. ${ }^{7,8}$ The need for molecular classification is for categorizing the patients who can benefit from targeted therapy (hormonal therapy and anti HER 2 therapy). ${ }^{7}$

Breast cancer with same histologic subtype may respond differently to therapy and may have different prognosis. Triple negative and HER 2 are more aggressive subtypes, with shorter survival period. Although they tend to respond better to chemotherapy. ${ }^{9}$ Also, triple negative breast cancers with tumor infiltrating lymphocytes have better prognosis and survival rate those without tumor infiltrating lymphocytes. ${ }^{10}$

\section{Materials and Methods}

This is a retrospective study. All female patients diagnosed with breast carcinoma at our institute between January 2019 and December 2019 were included. We divided the cases into two groups-group 1: $\leq 40$ years of age (younger group) and group 2: > 40 years (older group). Among them we studied histological type, grade, ER, PR, HER 2 status, and molecular classification.

\section{Immunohistochemistry}

Immunohistochemistry (IHC) was done on 4- $\mu$ m thick formalin fixed paraffin embedded tissue sections. Antigen retrieval was done using Tris-EDTA buffer and machine used was benchmark. Antibodies used for ER, PR, HER 2, and Ki67 were monoclonal antibodies against estrogen receptor (Clone SP1), progesterone receptors (Clone Y85), Her2 receptor (Clone SP3), and Ki 67 receptor (Clone SP6), respectively. Then percentage of cells staining positive was recorded.

\section{Guidelines for Immunohistochemistry Reporting}

According to ASCO and CAP guidelines, ${ }^{11,12}$ cutoff of $1 \%$ of tumor cells positive for nuclear stain of ER/PR was considered to be positive and the tumors staining $<1 \%$ of any intensity was considered negative. For HER 2, a semiquantitative scoring system called Allred scoring system was used which is based on percentage of positively stained cells and intensity of nuclear stain of the cells. HER 2 was scored from 0 to 3 .

0 : No stain or incomplete, faint in $<10 \%$ tumor cells.

$1+$ : Faint, incomplete staining in $<10 \%$ tumor cells.

$2+$ : Complete, weak to moderate staining in $>10 \%$ of tumor cells.

$3+$ : Complete circumferential membrane staining in $>10 \%$ of tumor cells.

The tumors were classified into four groups (luminal A, luminal B, HER 2, and triple negative) according to ER/PR/HER 2 status/Ki 67:

1. Luminal A: ER+ and/or PR+, HER 2-, Ki $67 \leq 14 \%$.

2. Luminal B: ER+ and/or PR+ and HER 2+ or if HER 2-then Ki $67>14 \%$.

3. HER 2: ER-, PR-, HER 2+.

4. Triple negative: ER-, PR-, HER 2-.

Out of total 568 cases, molecular typing could not be done in 189 cases due to either of these three reasons:

1. IHC was not available.

2. HER 2 borderline was not followed by FISH (fluorescence in situ hybridization).

3. Ki 67 was not available.

\section{Statistical Analysis}

Statistical analysis was done by using SPSS software version 20.0.

\section{Results}

Out of 568 cases studied, 151 cases (26.6\%) were of $\leq 40$ years age group while 417 cases $(73.4 \%)$ were $>40$ years age group with mean and median age 48.14 years and 47 years, respectively.

The most common histological subtype was ductal carcinoma in 548 cases (96\%) followed by lobular carcinoma in nine cases (1.5\%) and other $2.5 \%$ included cribriform carcinoma in four cases, two cases each of papillary carcinoma and mucinous carcinoma, one case each of carcinosarcoma, squamous cell carcinoma, and apocrine carcinoma ( - Table 1 ).

Among ductal carcinomas, the most common grade was grade III, seen in 278 cases (50.7\%) followed by grade II in 263 cases (48\%) (- Table 2 ).

Out of nine lobular carcinomas, seven were of grade I and two of grade II. Two out of four cribriform carcinoma had grade I and for another two, grade was not available. One out of two papillary and two mucinous carcinomas had grade I, for second cases in both grade was not available. Carcinosarcoma, squamous cell carcinoma, and apocrine carcinoma were not graded.

IHC was done for 432 cases (76\%) and Ki 67 was done in 299 cases (52.6\%). ER positivity was seen in 256 cases (59\%) and PR in 206 cases (47.7\%). HER 2 was positive in 104 cases (24\%), borderline in 88 cases $(20.4 \%)$. 
Table 1 Distribution of histological subtypes of breast carcinoma

\begin{tabular}{|l|l|}
\hline Histological type & Number \\
\hline Ductal carcinoma & $548(96.4 \%)$ \\
\hline Lobular carcinoma & $9(1.6 \%)$ \\
\hline Cribriform carcinoma & $4(0.7 \%)$ \\
\hline Papillary carcinoma & $2(0.35 \%)$ \\
\hline Mucinous carcinoma & $2(0.35 \%)$ \\
\hline Carcinosarcoma & $1(0.2 \%)$ \\
\hline Squamous cell carcinoma & $1(0.2 \%)$ \\
\hline Apocrine carcinoma & $1(0.2 \%)$ \\
\hline
\end{tabular}

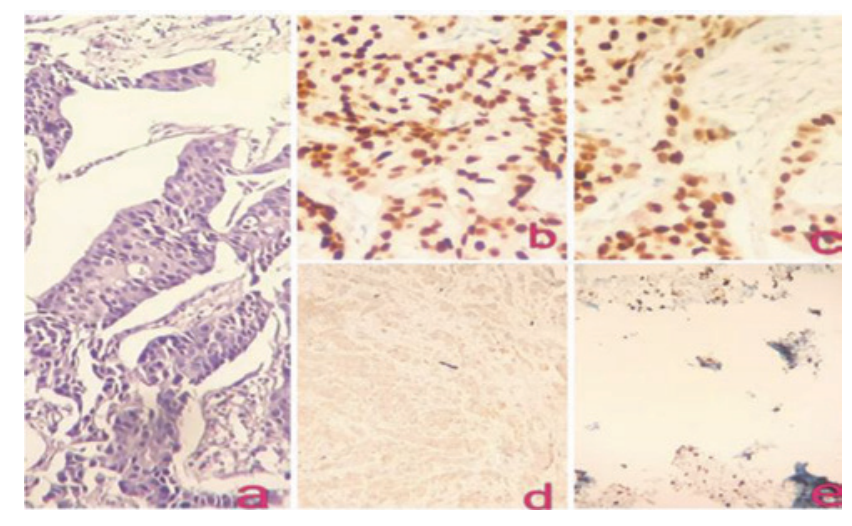

Fig. 1 Luminal A.

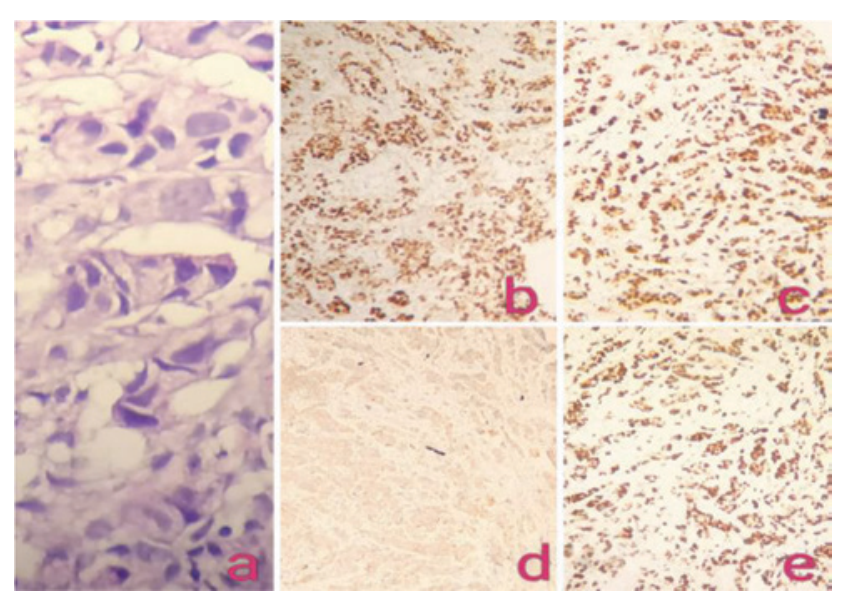

Fig. 2 Luminal B.

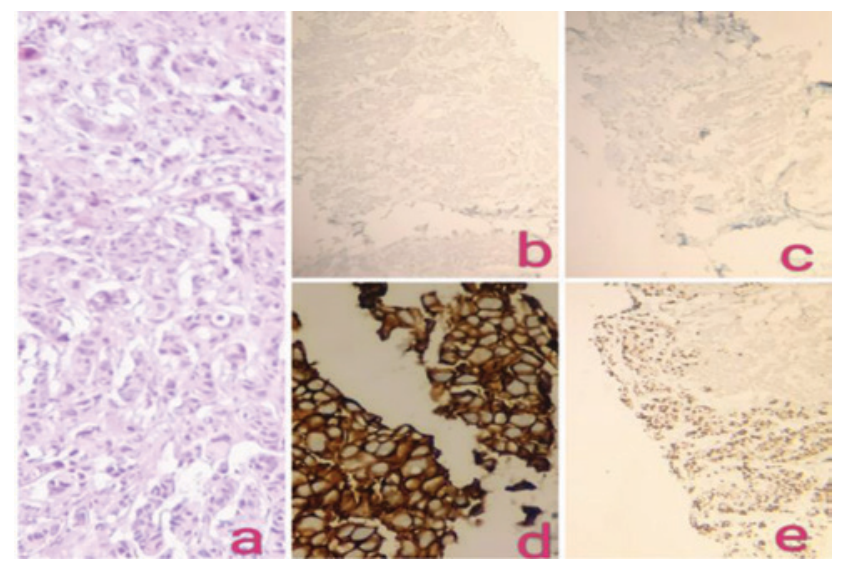

Fig. 3 HER 2.

and AlZaman et $\mathrm{al}^{4}$ where the mean age in younger age group was 37 and 36 years, respectively while in older age group the mean age was 54 and 55 years, respectively.

The most common histological subtype of breast carcinoma was ductal carcinoma similar to studies done by AlZaman et al, ${ }^{4}$ Alnegheimish et al, ${ }^{7}$ Goksu et al, ${ }^{13}$ and Kumar et al. ${ }^{8}$

ER positivity was seen in $59 \%$ and PR in $47.7 \%$. HER 2 positivity in $24 \%$, borderline in $20.4 \%$ cases, unlike in a study done by Alnegheimish et al. ${ }^{7}$ ER positivity was seen 
in $70.8 \%$ and PR in $63.8 \%$, HER 2 positivity in $18.7 \%$, and borderline in $22.8 \%$ cases.

The most common grade was grade III (50.7\%) followed by grade II (48\%), and grade I (1.3\%) unlike the study done by Engstrøm et al, ${ }^{6}$ where the most common was grade II (53.7\%) followed grade III (33.4\%), and grade I (12.9\%).

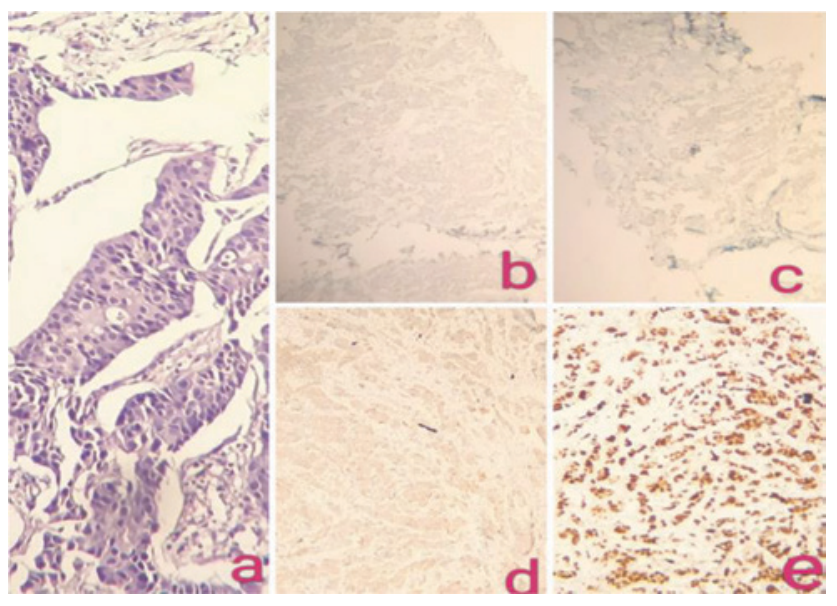

Fig. 4 Triple negative.
The most prevalent molecular subtype was luminal B followed by triple negative, luminal A and HER 2, unlike in a study done by Gupta et $\mathrm{al}^{1}$ and Lin et $\mathrm{al}^{3}$ in which luminal A was the most common followed by triple negative, HER 2, and luminal $\mathrm{B}$ type. In a study done by Alnegheimish et $\mathrm{al}^{7}$ the most common molecular subtype was luminal A followed by triple negative, luminal B, and HER 2 (- Table 5).

Two out of four cribriform carcinoma were of luminal A and other two of luminal B, six out of nine ILC were of luminal A and one each of luminal B, HER 2, triple negative. Both cases of mucinous and papillary carcinoma were of luminal A. Apocrine carcinoma, carcinosarcoma, and squamous cell carcinoma could not be typed.

Younger females had grade III tumors and luminal B as the most common molecular subtype similar to a study done by Lee et al. ${ }^{5}$

Luminal A had the highest proportion of grade II followed by equal proportion of grade I and grade III, HER 2 and triple negative had the highest proportion of grade III followed by grade II like a similar study done by Engstrøm et $\mathrm{al}^{6}$ according to which luminal A had the highest proportion of grade I and grade II while HER 2 and triple negative had the highest proportion of grade III followed by grade II.

Table 4 Distribution of molecular subtypes according to different grades

\begin{tabular}{|l|l|l|l|l|l|}
\hline Grade & Luminal A & Luminal B & HER 2 & Triple negative & Total \\
\hline Grade I & 17 & 3 & 0 & 0 & 20 \\
\hline Grade II & 37 & 86 & 22 & 26 & 265 \\
\hline Grade III & 17 & 74 & 37 & 60 & 277 \\
\hline
\end{tabular}

Abbreviation: HER, human epidermal growth factor.

Table 5 Prevalence of molecular subtypes of breast carcinoma among different population

\begin{tabular}{|l|l|l|l|l|}
\hline Author, Country & Luminal A & Luminal B & HER 2 & Triple negative \\
\hline Present study & $71(18.7 \%)$ & $163(43 \%)$ & $59(15.5 \%)$ & $86(22.8 \%)$ \\
\hline Gupta et al, (India) & $36(60.6 \%)$ & $2(3.3 \%)$ & $6(10.0 \%)$ & $16(26.7 \%)$ \\
\hline Lin et al, Taiwan (Western) & $635(62 \%)$ & $90(9 \%)$ & $121(12 \%)$ & $132(13 \%)$ \\
\hline $\begin{array}{l}\text { Alnegheimish et al Saudi } \\
\text { Arabia (Middle east) }\end{array}$ & $210(58.5 \%)$ & $52(14.5 \%)$ & $44(12.3 \%)$ & $53(14.8 \%)$ \\
\hline AlZaman et al, Bahrain (Asian) & $45(41.3 \%)$ & $24(22 \%)$ & $25(23 \%)$ & $15(13.7 \%)$ \\
\hline
\end{tabular}

Table 6 Comparison of age cutoff for younger and older age group breast carcinomas in different studies

\begin{tabular}{|l|l|l|}
\hline Author, Country & Age cutoff for younger age group & Age cutoff for older age group \\
\hline Present study & $\leq 40$ & $>40$ \\
\hline Gupta et all, India & $\leq 40$ & $>40$ \\
\hline Goksu et al, Turkey & $\leq 35$ & $>35$ \\
\hline Lin et al, Taiwan & $\leq 50$ & $>50$ \\
\hline AlZaman et al, Bahrain & $\leq 40$ & $>40$ \\
\hline
\end{tabular}


Different age cutoff values for younger and older age group are shown in - Table 6 .

\section{Conclusion}

Molecular classification is useful not only for prognosis, but also for the targeted therapy. Thus, it should be adopted as a part of routine histopathological reporting. Our study compared molecular subtypes of breast cancer and age in Northeast Indian women and luminal B is found to be the most common subtype as compared with other studies where luminal A was the most common subtype. This could be due to the reason that Ki 67 was not done in most of the other studies.

\section{Note}

This study was conducted at Dr. B. Borooah Cancer Institute, Guwahati, Assam. Total of 568 cases of breast cancer were taken and were classified into different molecular subtypes with the help of ER/PR/HER 2/Ki 67. Our study had advantage over other studies as Ki 67 was done and thus we got luminal $\mathrm{B}$ as the most common subtype unlike luminal A in other studies.

\section{Funding \\ None.}

\section{Conflict of Interest}

None declared.

\section{References}

1 Gupta P, Rai NN, Agarwal L, Namdev S. Comparison of molecular subtypes of carcinoma of the breast in two different age groups: a single institution experience. Cureus 2018;10(6):e2834

2 Sun YS, Zhao Z, Yang ZN, et al. Risk factors and preventions of breast cancer. Int J Biol Sci 2017;13(11):1387-1397
3 Lin CH, Liau JY, Lu YS, et al. Molecular subtypes of breast cancer emerging in young women in Taiwan: evidence for more than just westernization as a reason for the disease in Asia. Cancer Epidemiol Biomarkers Prev 2009;18(6):1807-1814

4 AlZaman AS, Mughal SA, AlZaman YS, AlZaman ES. Correlation between hormone receptor status and age, and its prognostic implications in breast cancer patients in Bahrain. Saudi Med J 2016;37(1):37-42

5 Lee MK, Varzi LA, Chung DU, et al. The effect of young age in hormone receptor positive breast cancer. BioMed Res Int 2015;2015:325715

6 Engstrøm MJ, Opdahl S, Hagen AI, et al. Molecular subtypes, histopathological grade and survival in a historic cohort of breast cancer patients. Breast Cancer Res Treat 2013;140(3):463-473

7 Alnegheimish NA, Alshatwi RA, Alhefdhi RM, Arafah MM, AlRikabi AC, Husain S. Molecular subtypes of breast carcinoma in Saudi Arabia. A retrospective study. Saudi Med J 2016;37(5):506-512

8 Kumar N, Patni P, Agarwal A, Khan MA, Parashar N. Prevalence of molecular subtypes of invasive breast cancer: a retrospective study. Med J Armed Forces India 2015;71(3):254-258

9 Rouzier R, Perou CM, Symmans WF, et al. Breast cancer molecular subtypes respond differently to preoperative chemotherapy. Clin Cancer Res 2005;11(16):5678-5685

10 Marra A, Viale G, Curigliano G. Recent advances in triple negative breast cancer: the immunotherapy era. BMC Med 2019;17(1):90

11 Hammond ME, Hayes DF, Dowsett M, et al. American Society of Clinical Oncology/College Of American Pathologists guideline recommendations for immunohistochemical testing of estrogen and progesterone receptors in breast cancer. J Clin Oncol 2010;28(16):2784-2795

12 Wolff AC, Hammond ME, Schwartz JN, et al; American Society of Clinical Oncology/College of American Pathologists. American Society of Clinical Oncology/College of American Pathologists guideline recommendations for human epidermal growth factor receptor 2 testing in breast cancer. Arch Pathol Lab Med 2007;131(1):18-43

13 Goksu SS, Tastekin D, Arslan D, et al. Clinicopathologic features and molecular subtypes of breast cancer in young women (age s35) Asian Pac J Cancer Prev 2014;15(16):6665-6668 